\title{
AGB variables in globular clusters
}

T. Lebzelter

University of Vienna, Türkenschanzstrasse 17, A1180 Vienna, Austria

P. Wood

RSAA, Australian National University, Canberra, Australia

K.H. Hinkle, R. Joyce

NOAO, Tucson, AZ 85726, USA

F. Fekel

Tennessee State University, Nashville, TN 37203-3401, USA

\begin{abstract}
With a large number of newly detected red variables in globular clusters we construct a $\log P-K$ diagram of these stars and compare it with findings from the LMC. We show the existence of a relation between the location in $\log P-K$ diagram and the velocity amplitude in the near infrared.
\end{abstract}

\section{Introduction}

While there has been a large effort to detect and classify variable stars in globular clusters, most search programs were designed to optimize detection and period determination for variables with periods less than one to a few days (e.g. Clement et al. 2001). AGB variables have therefore not been well surveyed and the existing sample is definitely far from complete. A large number of red variables has been detected but periods and amplitudes are missing for the vast majority of them (e.g. Pritzl et al. 2002). On the other hand, AGB variables in globular clusters offer a unique possibility to study AGB pulsation on stars that are well-defined in terms of luminosity, metallicity and mass. We therefore started an observing program to detect new AGB variables in globular clusters (GCs). Furthermore, we made a study on the pulsational properties of known long period variables in the globular cluster 47 Tuc.

\section{New AGB variables in globular clusters}

A photometric monitoring program of 12 globular clusters has been undertaken with the 50 in telescope at MSO between 2002 May and 2003 January. Additional data on 47 Tuc come from the YALO telescope at CTIO. Detection of variables was done with the help of the image subtraction tool ISIS 2.1 (Alard 
2000). Here we report on the current status of our project. We found a total of 102 variables with periods longer than 30 days, 67 of them detected as variables for the first time. Several of the new detections are located quite close to the cluster core where most previous search programs were saturating the image.

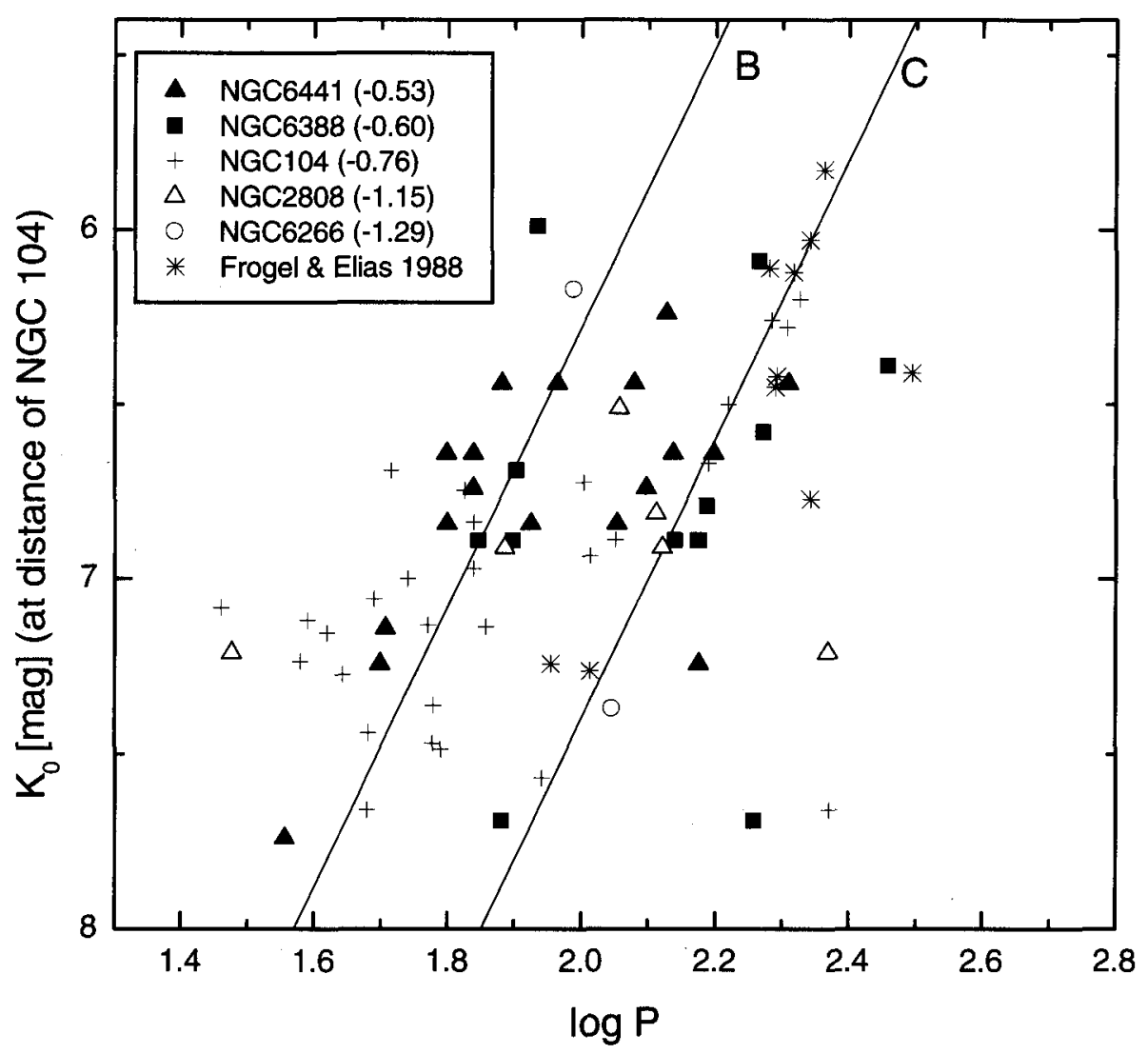

Figure 1. $\log P-K$ diagram for GC red variables. Different symbols denote variables from different clusters. In the legend numbers in brackets give the metallicity for each cluster. The approximate location of the two sequences B and C from Wood (2000) are indicated.

Fig. 1 shows a $\log P-K$ diagram of the red variables in the GCs analyzed so far. $K$ brightnesses have been taken from the 2MASS point source catalogue and shifted to the distance of 47 Tuc using distance moduli given in Rosenberg et al. (2000). Metallicities of the clusters are from Clement et al. (2002). Also given in this plot is the mean location of sequences $\mathrm{B}$ and $\mathrm{C}$ from the study on LMC red variables by Wood (2000). It can be seen that the red variables in GCs nicely fit on the two sequences. More metal-rich clusters show a lot more red variables than metal-poor ones, but still $\mathrm{AGB}$ variables can be detected down to metallicities of $[\mathrm{Fe} / \mathrm{H}]=-1.29$. 
Furthermore we mention the interesting case of NGC 6441. In contrast to the other clusters, variable stars occupy both sequences $\mathrm{B}$ and $\mathrm{C}$ to the same upper brightness limit. In other clusters (see also Fig. 2), the upper luminosity limit of stars on sequence B coincides with the lower limit of sequence $\mathrm{C}$. The outstanding behavior of NGC 6441 may indicate a wider mass spread in this cluster. Data reduction will be continued to establish a relation between the occurrence of AGB variables and metallicity as well as a better characterization of AGB pulsation in different clusters.

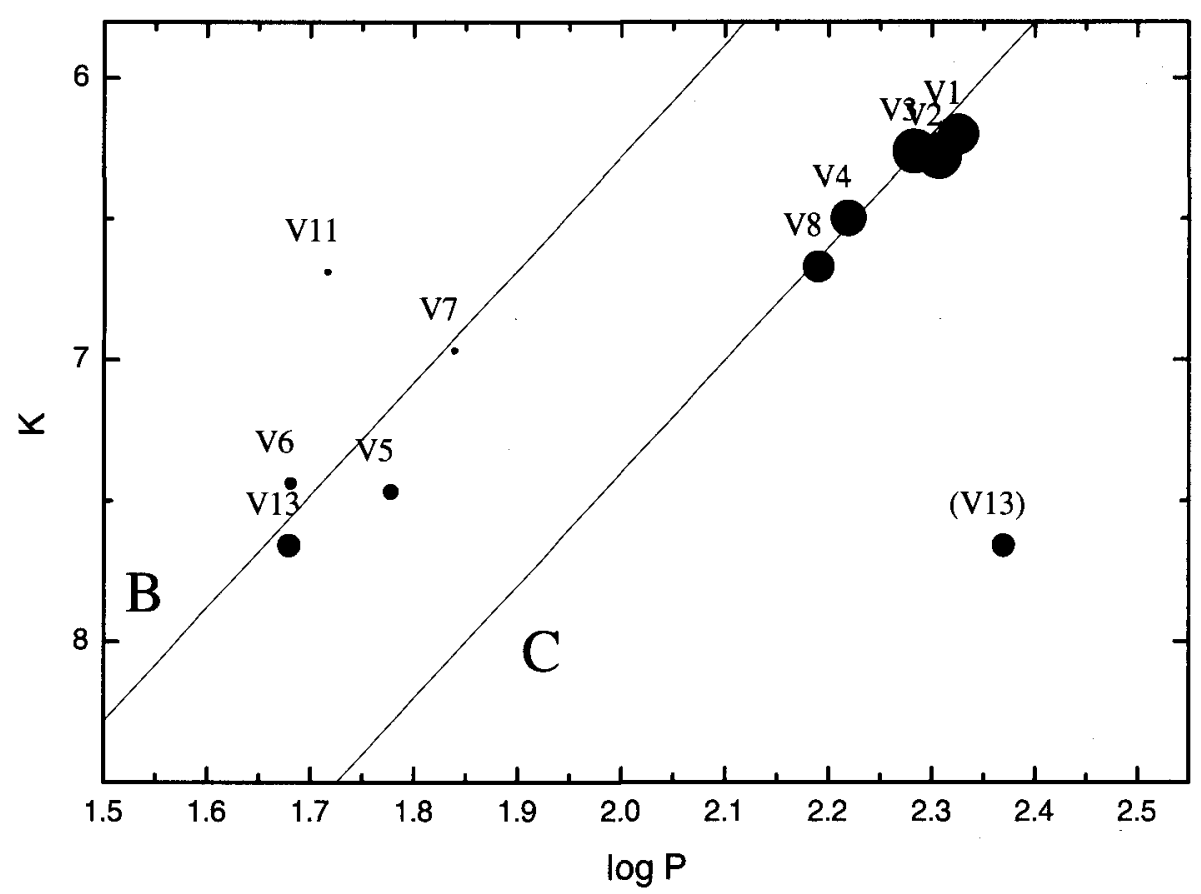

Figure 2. $\log P-K$ diagram for the long period variables in 47 Tuc. Symbol size indicates the NIR velocity amplitude (ranging from 4 to $23 \mathrm{~km} \mathrm{~s}^{-1}$. The approximate location of the two sequences $\mathrm{B}$ and $\mathrm{C}$ from Wood (2000) are indicated. See text for details.

\section{Velocity monitoring}

Time series of high-resolution, near-infrared spectra of ten AGB variables in the nearby metal-rich cluster 47 Tuc have been obtained using the 74 in telescope at MSO with the NICMASS detector. Observations span a total of 1.5 years. The near infrared lines of $\mathrm{CO}$ located in the $H$ and $K$ bands turned out to provide a powerful tool for studying the atmospheric kinematics of AGB stars (e.g. Lebzelter \& Hinkle 2002). Previous measurements were restricted to solar neighborhood AGB variables for which global parameters are very uncertain. For 
comparison with model atmospheres (e.g. Höfner et al. 2003) tight constraints on these parameters, which can be given for GC variables, are of critical importance.

The velocity changes measured in the 47 Tuc variables give comparable results to the findings in solar neighborhood LPVs. In Fig. 2 we give the location of the 47 Tuc variables in a $\log P-K$ diagram with the measured velocity amplitude for each star indicated. It can be seen that large velocity amplitude stars gather at the top of sequence C. Going down this sequence to lower luminosity, the velocity amplitude decreases. On sequence B we find only variables with small velocity amplitudes. The only exception is the star V13 which has a second long period (on Wood's sequence D?) dominating the velocity amplitude of the star.

\section{Outlook}

Our research on the occurrence of LPVs in GCs and their pulsational properties will be continued. The results will be compared with other features of these stars in relation to their location on Wood's pulsational sequences like mass loss, abundances of different elements and isotopic ratios. By deriving general properties of AGB stars in GCs we will give an important input for the understanding of AGB pulsation and mass loss in general as well as stellar evolution in GCs.

Acknowledgments. TL is receiving an APART grant from the Austrian Academy of Science. NOAO is operated by the Association of Universities for Research in Astronomy, Inc., under cooperative agreement with the National Science Foundation. This research has made use of the NASA/IPAC Infrared Science Archive. This publication makes use of data products from the Two Micron All Sky Survey.

\section{References}

Alard, C. 2000, A\&AS, 144, 363

Clement, C.M., et al. 2001, AJ, 122, 2587

Höfner, S., et al. 2003, A\&A, 399, 589

Lebzelter, T., Hinkle, K.H. 2002, in ASP Conf. Ser. Vol. 259, Radial and nonradial pulsations as probes of stellar physics, ed. C. Aerts, T.R. Bedding \& J.C. Christensen-Dalsgaard (San Francisco ASP), 556

Pritzl, B.J., et al. 2002, AJ, 124, 949

Rosenberg, A., et al. 2000, A\&AS, 144, 5

Wood, P.R. 2000, PASA, 17, 18 


\section{Discussion}

Alves: Can you explain how you classify your stars as being on the AGB as opposed to, for example, being on the first-ascent RGB?

Lebzelter: Those brighter than the tip of the RGB are AGB stars, while those fainter are RGB stars.

Bono: Are the LPV stars in NGC 6441 and NGC 6338 selected on the basis of solid velocity measurements?

Lebzelter: No. Membership in the cluster was assumed from location close to the cluster centre and luminosity/location in the HR Diagram.

Catelan: In our HST snapshot survey of NGC 6441, we have detected 12 LPVs, encompassing $\sim 2.5 \mathrm{mag}$ in $V$ (Pritzl et al. 2003, AJ, in press; Astro-Ph/0305339).

Lebzelter: Very interesting, thank you.

Dambis: What is the period ratio for sequences B and C?

Lebzelter: The two sequences in the plot mark the approximate location of the two sequences $\mathrm{B}$ and $\mathrm{C}$ from the MACHO LMC date. They are not a fit to the 47 Tuc data. As seen in the MACHO data there is some scatter around these sequences. The two lines in my plot are separated by $\log P=0.27$.

Habing: Brief comment: Some stars with luminosity below the RGB will be AGB - the majority will be RGB, but the LPVs among them might still be AGB.

Lebzelter: I agree.

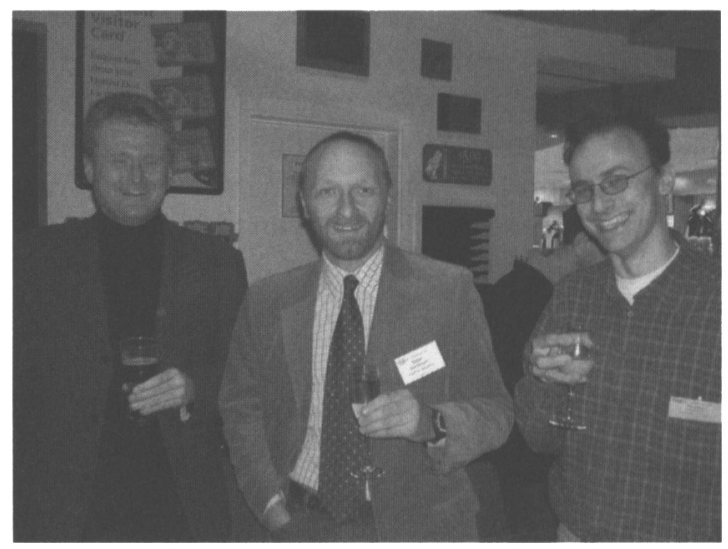

Gerald Handler, Franz Kerschbaum and Thomas Lebzelter 